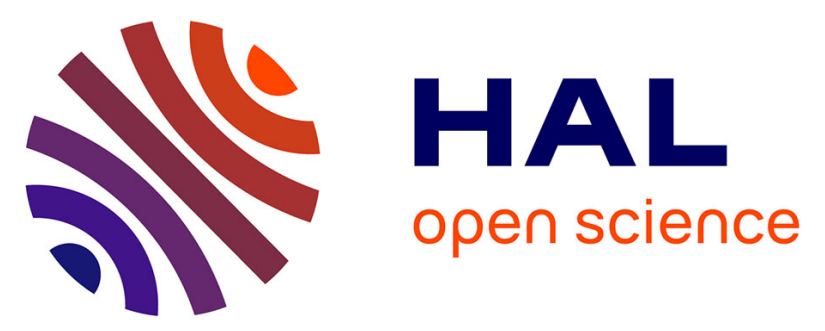

\title{
Model-Based Analysis-Synthesis for Realistic Tree Reconstruction and Growth Simulation
}

Corina Iovan, Paul-Henry Cournède, Thomas Guyard, Benoît Bayol, Dider Boldo, Matthieu Cord

\section{- To cite this version:}

Corina Iovan, Paul-Henry Cournède, Thomas Guyard, Benoît Bayol, Dider Boldo, et al.. Model-Based Analysis-Synthesis for Realistic Tree Reconstruction and Growth Simulation. IEEE Transactions on Geoscience and Remote Sensing, 2014, 52 (2), pp.1438-1450. 10.1109/TGRS.2013.2251467 . hal00826023

\section{HAL Id: hal-00826023 \\ https://hal-centralesupelec.archives-ouvertes.fr/hal-00826023}

Submitted on 25 May 2013

HAL is a multi-disciplinary open access archive for the deposit and dissemination of scientific research documents, whether they are published or not. The documents may come from teaching and research institutions in France or abroad, or from public or private research centers.
L'archive ouverte pluridisciplinaire HAL, est destinée au dépôt et à la diffusion de documents scientifiques de niveau recherche, publiés ou non, émanant des établissements d'enseignement et de recherche français ou étrangers, des laboratoires publics ou privés. 


\title{
Model-Based Analysis-Synthesis for Realistic Tree Reconstruction and Growth Simulation
}

\author{
Corina Iovan, Paul-Henry Cournède, Thomas Guyard, Benoît Bayol, Didier Boldo, and Matthieu Cord
}

\begin{abstract}
Due to complexity, vegetation analysis and reconstruction of remote sensing data are challenging problems. Using architectural tree models combined with model inputs estimated from aerial image analysis, this paper presents an analysissynthesis approach for urban vegetation detection, modeling, and reconstruction. Tree species, height, and crown size information are extracted by aerial image analysis. These variables serve for model inversion to retrieve plant age, climatic growth conditions, and competition with neighbors. Functional-structural individual-based tree models are used to reconstruct and visualize virtual trees and their time evolutions realistically in a 3-D viewer rendering the models with geographical coordinates in the reconstructed scene. Our main contributions are: 1) a novel approach for generating plant models in 3-D reconstructed scenes based on the analysis of the geometric properties of the data, and 2) a modeling workflow for the reconstruction and growth simulation of vegetation in urban or natural environments.
\end{abstract}

Index Terms-Dynamic scene evolution, functional-structural tree growth model, image-based vegetation modeling, species classification, vegetation detection.

\section{INTRODUCTION}

$\mathbf{U}$ NTIL recently, trees or vegetation have merely been considered as contextual elements in virtual environments. They are generally used to add some visual realism to the virtual scene. For this purpose, gifted computer graphists can provide some very beautiful and satisfying results [1], [2].

However, with the increasing environmental concern [3], virtual trees and plants get growing attention and their biophysical realism may be as important as their visual realism for specific applications, in both urban and natural contexts. Particularly, one may be interested in the dynamical evolution of the virtual environment. For example, for urban applications, we may be interested in visualizing what the evolution

Manuscript received May 16, 2012; revised February 10, 2013; accepted February 12, 2013.

C. Iovan is with INRIA Saclay - Île-de-France (the French Institute for Research in Information and Communication Science and Technologies), Saclay, Ile de France 91120, and also with the French National Survey Agency, Palaiseau 91120, France (e-mail: corina.iovan@gmail.com).

P-H. Cournède is with the MAS Laboratory, Ecole Centrale Paris, Paris 92295, France (e-mail: paul-henry.cournede@ecp.fr).

T. Guyard and B. Bayol are with INRIA Saclay - Île-de-France (the French Institute for Research in Information and Communication Science and Technologies), Saclay, Ile de France 91120, France (e-mail: thomas.guyard@inria.fr; benoit.bayol@inria.fr).

D. Boldo was with the French National Survey Agency, Saint-Mandé 94165, France (e-mail: didier.boldo@edf.fr).

M. Cord is with the UPMC Paris 6 - Sorbonne Universités, Paris 75005, France (e-mail: matthieu.cord@lip6.fr).

Color versions of one or more of the figures in this paper are available online at http://ieeexplore.ieee.org.

Digital Object Identifier 10.1109/TGRS.2013.2251467 of a park will be after a few years. For forestry applications, it is crucial to predict the growth of a population of trees, according to scenarios of exploitation. In both cases, the virtual environment should also help answering some scientific questions, e.g., what will be the consumption of carbon dioxide of trees in the city/forest over the years [4], [5]?

Canopy reconstruction algorithms have known great interest as a useful tool for forest ecosystem studies [6]. Understanding the backscattering processes in forest canopies is useful for monitoring vegetation canopies, and it potentially provides insight on the type of forest or processes driving its development. To this end, several models are designed to simulate the radar backscatter response for a variety of forest stands [7], [8].

In this context, the aim of this paper is to reconstruct functional vegetation in virtual environments, both geometrically and biophysically by using model-based analysis-synthesis approach. More specifically, we consider the case of 3-D city models including vegetation. A set of parameters for individual trees in a scene are estimated by image analysis from aerial data. They are used as inputs for a discrete dynamic system to generate functional-structural tree models for visualization and growth simulation. Moreover, the dynamic model of tree growth can be used to visualize the time evolution of the scene.

The methodology is based on recent progress in the characterization of vegetation from aerial image analysis [9] and in functional-structural plant growth models, which combine the description of both architectural development (and thus plant geometry) and functional growth, in interaction with the environment [10].

The analysis of aerial data aims at identifying vegetation characteristics. The system extracts vegetation areas, separates them into high- and low-height vegetation, delineates individual tree crowns, extracts 3-D tree parameters (such as crown area and tree height) and classifies them according to their species. With this information, the objective is thus to reconstruct all trees in the scene, from the functional-structural individual-based model of tree growth. The parameters specific to each species are known a priori, as they are estimated through specific experiments, mainly conducted by biologists. The missing pieces of information are tree ages, and conditions of growth (environmental, competition conditions). For each species, we perform a minimization of the distance between tree model simulation outputs and analysis results regarding height and crown size. From the model thus parameterized, we obtain the geometry of all plants and produce corresponding graphic models to be used for visualization in the 3-D city model. 


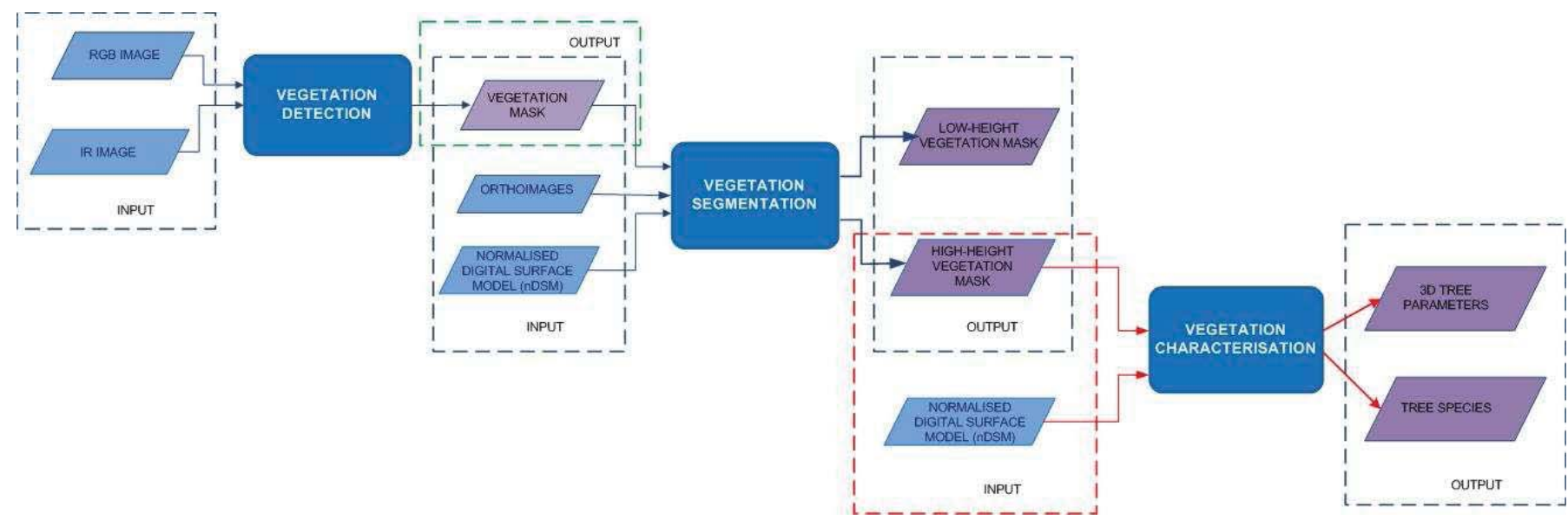

Fig. 1. Urban vegetation analysis-system overview. The first module extracts vegetation areas from high-resolution aerial data of urban areas. Vegetation masks are processed by the following module which separates them into short and tall vegetation areas and performs individual tree crown delineation. The last module is used to estimate 3-D parameters of trees and to classify them according to their species.

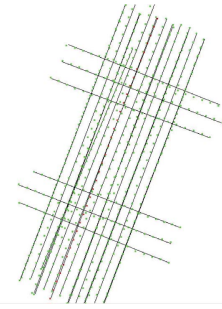

(a)

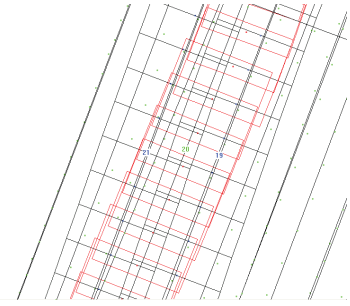

(b)

Fig. 2. Flight plan for the 2004 aerial-image acquisition campaign for the city of Marseille. (a) Total of 23 flight-strips are flown, six of which are perpendicular. Dots on each flight strip: the position where an image is captured. (b) Enlarged detail of flight strips 19-21 illustrating the 60\% overlap between images taken on the same strip and $60 \%$ between two adjacent strips. Areas covered by images taken in each strip is presented by a bounding rectangle.

This paper starts by presenting image analysis from aerial data and estimation of model inputs in Section II. Section III gives an overview of an individual-based architectural model of tree growth combining both structural development and biophysical functioning. The methodology for model parameterization from aerial imagery is detailed in Section IV. Experimental results are presented in Section V. Finally, conclusions and perspectives of our work are presented in Section VI.

\section{Aerial Data Analysis}

This section presents urban vegetation analysis from highresolution aerial imagery. Part of the works presented hereafter are presented in [9] and will be briefly summarized in the following. For a detailed description of the entire system, please refer to the aforementioned publication.

The approach to analyze vegetation from high-resolution aerial imagery is a hierarchical one: regions of interest containing vegetation are extracted in a first step and category of objects present therein are analyzed in the following steps either through segmentation or classification approaches. Fig. 1 shows the whole system for vegetation detection and characterization from aerial images.

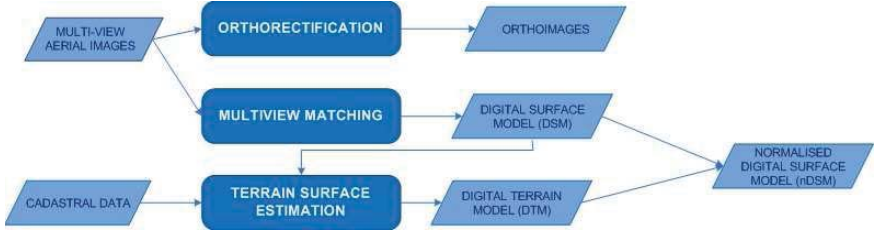

Fig. 3. Overview of the methodological process for dataset creation and data samples. Aerial images and cadastral data are used to obtain additional data. Due to the overlap between aerial images, the DSM obtained is accurate enough for 3-D object analysis.

\section{A. Data and Study Area}

The input of the system represents standard types of data produced for 3-D city modeling applications, such as high-resolution georeferenced aerial images with a resolution of $20 \mathrm{~cm}$ per pixel and having four channels [red (R), green (G), blue (B), and near-infrared (IR)].

The aerial images are acquired according to a flight plan (see Fig. 2) containing flight strips ensuring an overlap of $60 \%$ between images taken within each strip and $60 \%$ between two adjacent strips. This ensures that all the points of the studied area are visible on at least four to nine images. The chargedcoupled device matrix is made up of more than 16 million pixels $(4096 \times 4096)$ each having a size of $9 \mu \mathrm{m}$ [11]. The four channels (R, G, B, and IR) are acquired simultaneously by four different acquisition heads of a four-head camera system [12]. All aerial images are acquired the same day and preprocessed prior to vegetation extraction by correcting radiometric errors (haze reduction) and distortions of digital images. Radiometric corrections address variations in the pixel intensities that are not caused by the object or scene being scanned. Atmospheric haze reduction is a simple method that assumes that some image pixels should have a reflectance of zero [13]. Actual values of zero pixels result from atmospheric scattering. The method employed for haze correction consists in subtracting the histogram offset from all pixels in a specific band.

The flow chart presented in Fig. 3 shows the process of obtaining additional data from multiview aerial imagery. Fourchannel orthophotographs are obtained by removing the effects of image perspective and relief and creating a planimetrically 

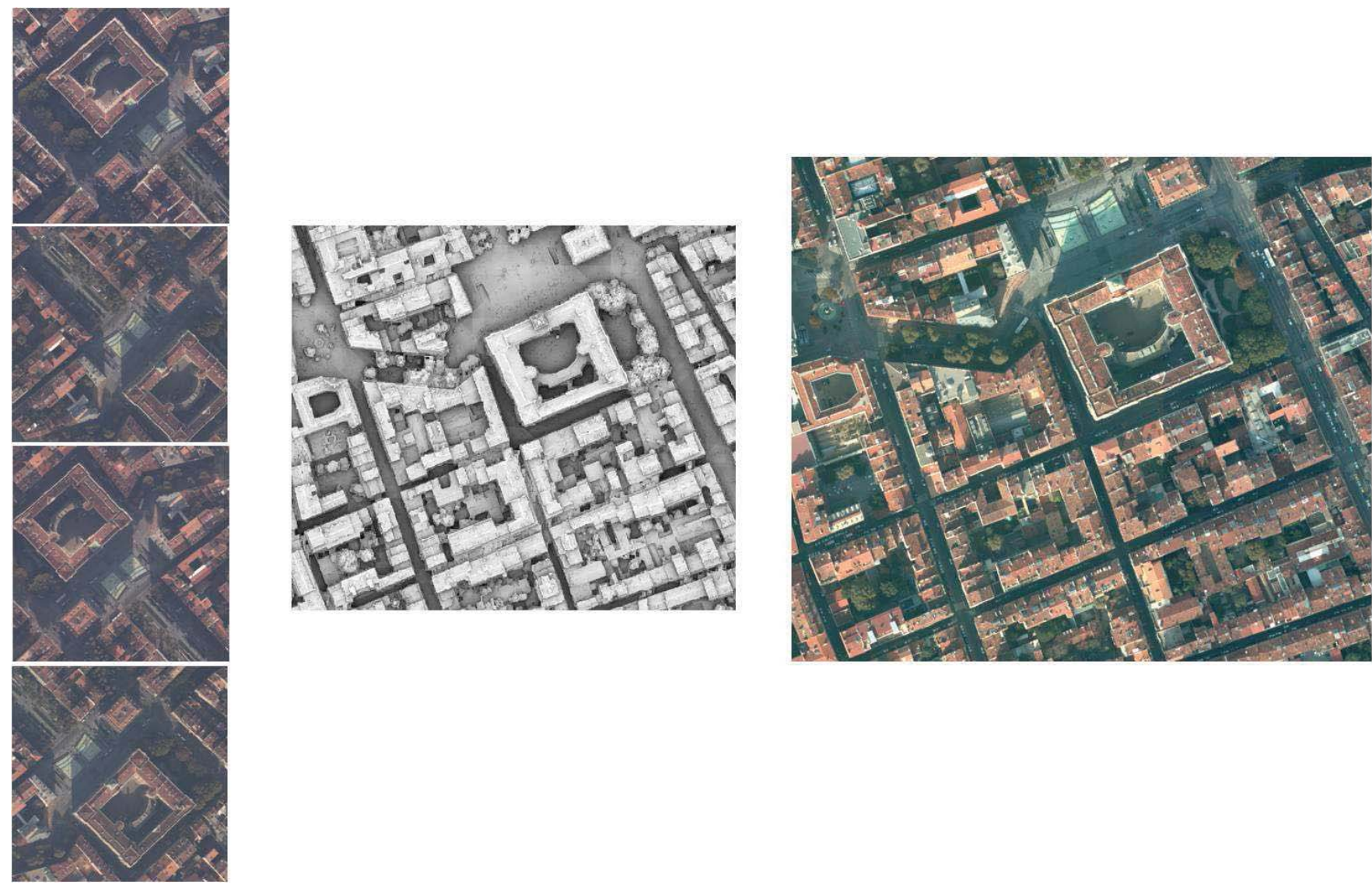

(a)

(b)

Fig. 4. Sample data acquired over the city of Marseille in 2004. (a) Set of four images from two neighbor flight strips in which the same building is visible. There are high-resolution RGB images in a conique perspective. The DSM obtained by correlation from the multiview images. (b) Resulting orthophotograph of the same area.

correct image, having a constant scale wherein features are depicted in their true plan positions. A dense digital surface model (DSM) is computed from multiple images using MICMAC (http://www.micmac.ign.fr) a multiview matching algorithm [12]. Buildings are masked on the DSM, and the resulting depth map is subsequently used to estimate the terrain surface, thus obtaining a digital terrain model (DTM) [14] which is a digital representation of the topographic surface. A normalized digital surface model (nDSM), containing the height of above ground objects, is computed as the difference between DSM and DTM. Sample data (aerial images, DSM, and orthophotograph) for the scene of Marseille are depicted in Fig. 4.

The study area is located in the city of Marseille, situated in the southeast of France. Marseille's climate is Mediterranean, with a great variety of vegetation species. It is a complex urban area, with many green resting places, highly intermingled with buildings. Fig. 5 depicts the location of the study area used in this paper.

\section{B. Vegetation Detection}

Traditionally, vegetation indexes such as the normalized difference vegetation index (NDVI) [15] are used to extract vegetation areas from remote sensing imagery. Although such indexes are developed for different applications and particular

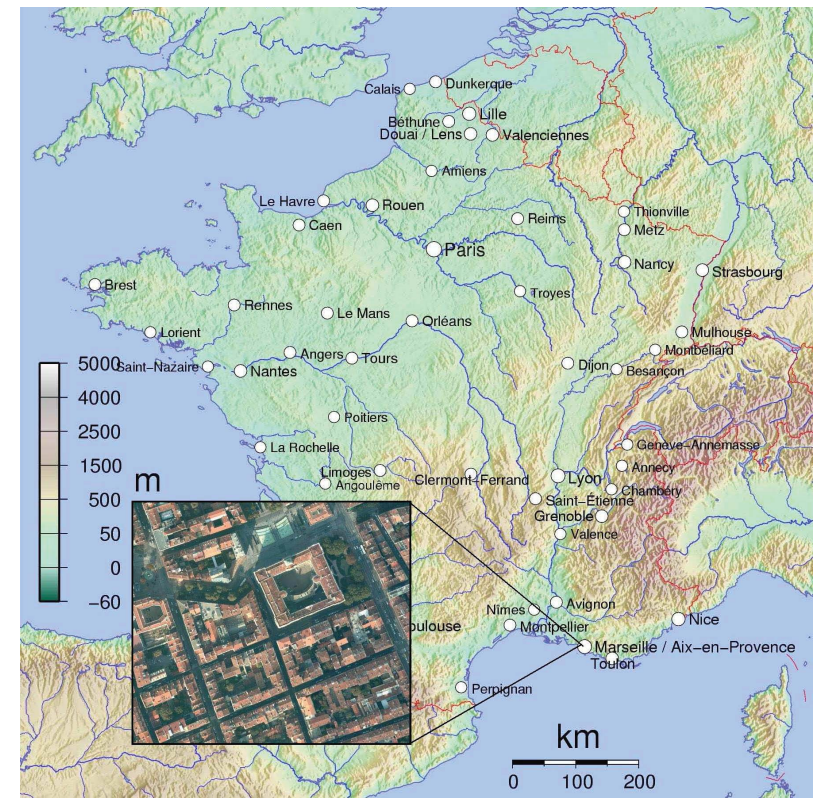

Fig. 5. Study area is located in the south of France, city of Marseille.

types of input data and acquisition conditions (which can be incompatible with the urban environment), there is no ideal index designed to characterize the urban environment [16]. The urban environment is a mixture of different landuse/land-cover classes. Therefore, the spectral response of 


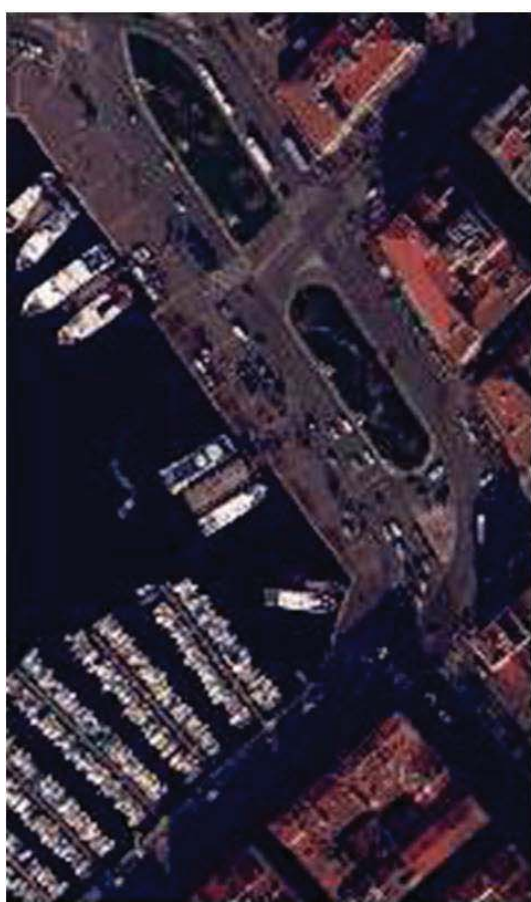

(a)

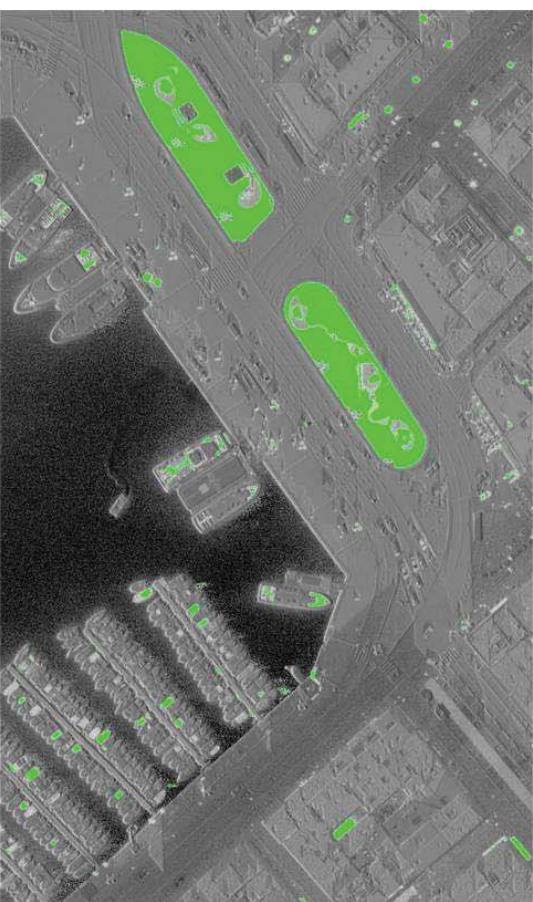

(b)

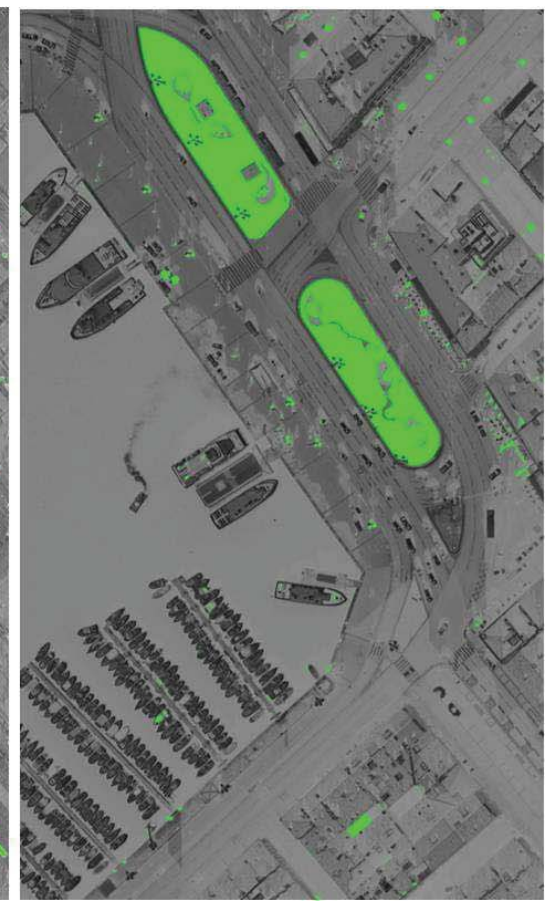

(c)

Fig. 6. Results for the vegetation detection step. (a) Original RGB image. (b) NDVI image. Green pixels: vegetation areas. (c) SVM classifier, linear kernel, and feature vector computed on the Lab color space.

urban vegetation is altered by the presence of different landuse/land-cover classes such as lawns, shrubs, and treed areas having similar spectral signatures. Moreover, the atmospheric conditions over urban areas are greatly influenced by the presence of pollutants and dust issued from industrial plants, which are mainly located around big cities. All these factors induce great variations in the spectral reflectance of the same urban material.

To overcome the limitations of such methods, we use a supervised classification approach based on spectral features and a support vector machines (SVMs) [17] classifier to extract vegetation in urban areas. This task is seen as a binary classification problem, where SVMs are used to learn from a set of labeled examples a classification rule, which should be used to predict the class of new unlabeled examples. Geometrically, this rule could be expressed as the hyperplane that separates the input space into two half-spaces, and the prediction of the class of a new point depends on the position of this point on one or the other side of the hyperplane. SVMs learn a classification function that, among all separating hyperplanes, maximizes the distance to the closest data point from the training data set.

The discriminative power of SVMs can be enriched via kernel functions, which allow the mapping of data points from the original space to another space, where linear classification between data sets might be possible. As the kernel defines a distance between data points, it can be seen as a measure of similarity between them: the larger the kernel, the closer data points are in the initial feature space. Several kernels are analyzed: linear, polynomial and radial basis function (RBF). For the classifier implementation, the LIBSVM [18] library is used.
TABLE I

Size of Training and Test Data Sets for Vegetation Detection

\begin{tabular}{|c|c|c|}
\cline { 2 - 3 } \multicolumn{1}{c|}{} & Training & Test \\
\cline { 2 - 3 } \multicolumn{1}{c|}{} & No. pixels & No. pixels \\
\hline Vegetation areas & 100000 & 250000 \\
\hline Nonvegetation areas & 100000 & 1000000 \\
\hline TOTAL & 200000 & 1250000 \\
\hline
\end{tabular}

Reflectance values of pixels in the IR, R, G, and B channels are used to compose the feature vector used by the SVM classifier during training. Several color transformations are applied to the R, G, and B components of the feature vector. Components of the hue, saturation, and value (HSV), XYZ and Lab color spaces are used in addition to the IR channel to perform vegetation detection.

Training and tests are performed on manually delineated samples of vegetation and nonvegetation areas. Table I gives the total number of pixels used for training and test. Training is performed on the same amount of pixels for the vegetation and nonvegetation classes to build reliable models. As for the test data set, the differences between the sizes of the two classes comes from the absence of enough ground truth pixels to test models on larger data sets.

Vegetation detection performances for the SMV classifier are given bellow by means of true positives (TP) rates for three false positives (FP) cases: $\mathrm{FP}=0.0001, \mathrm{FP}=0.001, \mathrm{FP}=0.01$, meaning, respectively, that one pixel out of 10000,1000 , and 100 is misclassified.

Table II emphasizes the great potential of the SVM-based approach for vegetation detection in urban areas compared with the traditional NDVI-based approach. For a fixed FP rate of 0.0001 , i.e., one out of 10000 pixels is misclassified, 
TABLE II

RESULTS FOR VEGETATION DETECTION BY SVM CLASSIFIER AND Comparison With the NDVi. Feature Vectors ARe Computed in DifFerent Color Spaces. TPS Rate Is Presented For THREe Values of FPS Rate

\begin{tabular}{|c|c|c|c|c|}
\hline Color Space & Kernel & FP $=1 \mathrm{e}-4$ & FP $=1 \mathrm{e}-3$ & $\mathrm{FP}=1 \mathrm{e}-2$ \\
\hline NDVI & - & 0.0882 & 0.907 & $\mathbf{0 . 9 9 8}$ \\
\hline RGB & linear & 0.57 & 0.903 & 0.997 \\
\hline RGB & poly & 0.848 & $\mathbf{0 . 9 9 3}$ & 0.997 \\
\hline RGB & RBF & 0.498 & 0.811 & 0.981 \\
\hline HSV & linear & 0.539 & 0.76 & 0.979 \\
\hline HSV & poly & $\mathbf{0 . 8 6 7}$ & 0.99 & 0.994 \\
\hline HSV & RBF & 0.467 & 0.939 & 0.988 \\
\hline XYZ & linear & 0.398 & 0.661 & 0.792 \\
\hline XYZ & poly & 0.467 & 0.696 & 0.816 \\
\hline XYZ & RBF & 0.397 & 0.649 & 0.776 \\
\hline Lab & linear & 0.629 & 0.991 & $\mathbf{0 . 9 9 8}$ \\
\hline Lab & poly & 0.859 & 0.98 & 0.994 \\
\hline Lab & RBF & 0.678 & 0.967 & $\mathbf{0 . 9 9 8}$ \\
\hline
\end{tabular}

performances of the NDVI-based approach are inferior to performances of all other SVM-based approaches for vegetation detection. For an FP rate of 0.001, the SVM-based approach still outperforms the NDVI one, by an approximately 9\% increase of the TP rate for the RGB color space and a polynomial kernel. For FP rates of 0.01, the NDVI-based approach performs equally to the SVM-based one, for the Lab color space with inear or RBF kernels.

Fig. 6 shows experimental results of the vegetation detection strategy proposed here and compares it with the traditional NDVI.

The linear kernel gives very good results for the feature vector computed in the Lab color space, which could be the result of the noninear relationships among the $\mathrm{L}$, $\mathrm{a}$, and $\mathrm{b}$ components of the color space as they intend to mimic the logarithmic response of the eye. The polynomial kernel gives equally good performances for the RGB and the Lab color spaces whereas the RBF kernel gives best performances on the Lab color space.

\section{Vegetation Segmentation}

The results of the first module are used as input for the vegetation segmentation module, constructed around a twostep strategy, starting with lawn delineation and continuing with individual tree crown delineation. Given the vegetation mask previously obtained and the DSM, height is the main source of information exploited to separate lawns from trees, and to individually separate tree crowns. As the aim of the first step is to distinguish between two spectrally close classes (lawn areas and trees), texture characteristics computed on the DSM are exploited to differentiate between grass and trees. The local height variance is computed on the vegetation areas corresponding to the DSM to extract lawns from the vegetation areas previously delineated. This texture feature accentuates large changes in height values between adjacent pixels. The results of simple thresholding techniques are on the basis of these features.

The methodology for lawn delineation can be resumed by the following steps:

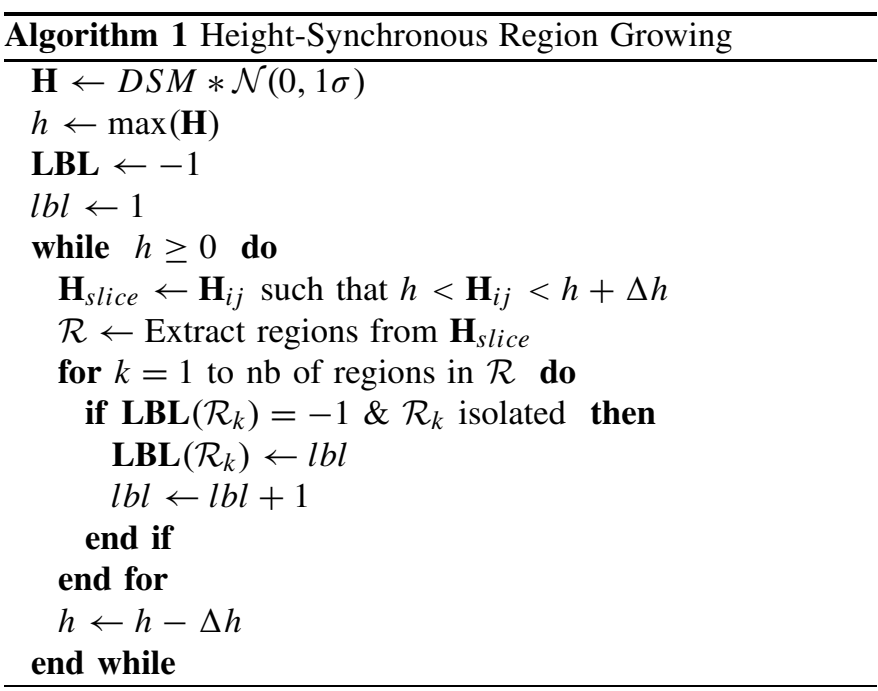

1) the DSM of the area is used to compute variance;

2) the vegetation mask is used on the image obtained after the first step to hide all nonvegetation areas;

3) the resulting image is passed on to a decision module which separates lawns from trees;

4) the output is saved as binary images representing lawn and tree areas.

Height local variance is computed by using a sliding window of an empirically determined size of $11 \times 11$ pixels. This settings allowed us to capture both fine-scale and coarser-scale height characteristics of urban vegetation. The variance texture data is separated into low- and high-values using a histogrambased thresholding method.

Provided an accurate DSM is available, the method gives reliable results for separating lawn areas from trees. In the context of our application, experimental results obtained by applying this method to separate trees from lawns proved to be highly satisfactory. At the output of the lawn delineation step, two thematic classes are isolated from the vegetation class. The first one contains lawn areas and the second, trees.

Tree crown delineation problems are tackled through topdown or bottom-up approaches. Top-down methods include an input model for tree crowns [19] into the segmentation algorithm, in contrast to bottom-up approaches, which aim to group pixels according to feature similarities such as continuity of pixel intensities [20], [21], texture [22], and bounding contours [23]. To delineate individual tree crown boundaries, we use a region growing approach, which belongs to the class of bottom-up methods and exploits tree height information from the DSM. It overcomes the drawback of topdown approaches on a priori knowledge of tree-crown shapes needed to generate the model. It consists of two steps. In the first one seed regions, corresponding to tree tops, are extracted from height information from the DSM. Algorithm I is used for seed extraction.

First the DSM is smoothed by a 2-D Gaussian filter, and the result is stored in $\mathbf{H}$. Then a local maximum search is performed and pixels corresponding to seed regions are labeled in the LBL image. These seed regions are grown in a second 


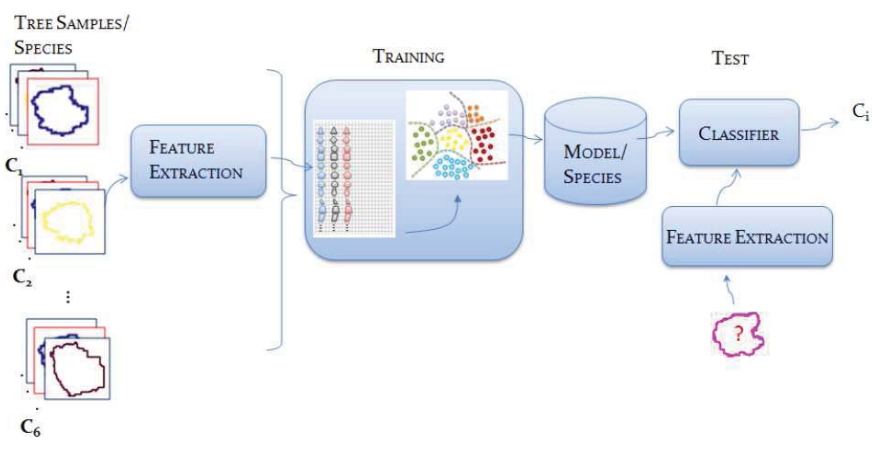

Fig. 7. System overview for tree species classification.

step by a watershed [24] like algorithm in which the growing process of a region stops when it connects to another region. The output of the vegetation segmentation module consists of individual regions corresponding to each tree crown, which will be exploited by the vegetation characterization module for both identifying morphological characteristics of trees and to perform tree species classification.

\section{Vegetation Characterization}

If the individual tree crown regions are obtained by the segmentation module, image data, and height information, the first component of the vegetation characterization module extracts morphology attributes for each tree crown. The second component, deals with tree species classification through supervised classification techniques. This is performed by using ground truth data of tree species, tree crown regions and spectral, and spatial characteristics computed for each individually delineated tree region.

1) Tree Crown Diameter $c d$ and Tree Height $h$ are estimated for each tree $T$ from regions $R_{i}$ obtained after tree crown delineation. The diameter of a crown $(c d)$ is estimated by measuring the length along one axis from edge to edge through the region's center. Tree height $h$ is estimated for each region from the $\mathrm{nDSM}$.

2) Tree Species are identified by an SVM-based supervised classification approach using spectral and texture features extracted from image data for each individual tree crown. The system used to perform tree species classification is illustrated in Fig. 7.

Input tree crown samples of each tree species (class) are used to extract spectral and spatial features which will form the feature vector for the training stage. During training, feature vectors belonging to one species are used to find the boundary separating all samples belonging to the class taken in consideration from all other samples not belonging to the class. During the test stage, features of an unknown tree sample are compared with models computed for each species during the training stage. The sample is assigned to the class giving the highest membership probability.

Let $R_{1}, R_{2}, \ldots, R_{N}$ denote tree crown regions in the segmented image $i(x, y)$. To classify the regions, feature vectors composed of spectral and texture measures are computed on whole tree crown samples $R_{i}$.

The distribution of reflectance values of pixels belonging to a tree crown region $R_{i}$ are modeled by a multivariate Gaussian
TABLE III

Tree Species Data Set: Species Names and Number of Samples

\begin{tabular}{lccc}
\hline Scientific Species Name & Samples & Train & Test \\
\hline Platanus sp. & 69 & 35 & 34 \\
Sophora japonica L. & 32 & 16 & 16 \\
Tilia sp. & 83 & 42 & 41 \\
Celtis australis L. & 29 & 15 & 14 \\
Pinus pinea L. & 36 & 18 & 18 \\
Cupressus sempervirens L. & 18 & 9 & 9 \\
\hline
\end{tabular}

model with full covariance matrix $\mathcal{N}(\mu, \Sigma)$. The spectral feature vector thus obtained is concatenated with a texture feature vector, composed of second-order statistics of the texture of each tree crown region $R_{i}$ extracted from the gray level cooccurrence matrix [25], namely mean, standard deviation, range, angular second moment, contrast, correlation, entropy, and inverse difference moment. The choice of an appropriate distance $d$ between pixels is closely related to the coarseness or the fineness of the texture being analyzed. The coarser the texture, the more the distance between pixels can be increased. Distance is set to $d=1$ to characterize texture in its finest level of detail. Direction is important in case of anisotropy in the texture. This is not the case for tree crowns. Thus, second-order statistics are computed over a direction of $0^{\circ}$. Both spectral and texture features are computed for each channel of different color spaces RGB, XYZ, Lab, and HSV [26], as well as the IR channel.

A linear SVM classifier [18] is trained by using a feature vector obtained by concatenating the spectral and the texture feature vectors. The discriminative SVM classifier is used in "one-against-all" configuration. Feature vectors are used in the training phase to build a set of binary classifiers able to separate each class from all others. This method trains $s$ SVMs (where $s$ is the number of classes which corresponds to tree species in the study area) and there are $s$ decision functions. In this paper, $s=6$. An unknown tree sample $T$ is classified as belonging to the class for which the largest decision value is determined [27].

Table III presents the division of the samples per group and species. The total of 267 tree crown contours is randomly divided into training and test subsets. Half of the total number of samples for each class is used to train the SVM classifier while the other half is used for tests.

Classification accuracy varies with the type of input features. Results obtained for tree species classification by using spectral and texture features, are presented in Table IV for the best combination of feature vectors and color spaces, evaluated in terms of overall accuracy (\%) (as the number of classes is higher than two). Best results are obtained when combining spectral features extracted on RGB color space with texture features computed on the HSV color space.

Fig. 8 illustrates tree species classification results obtained for tree crown areas delineated by the algorithm presented in the previous step.

The confusion matrix obtained for tree species classification is given below for spectral features computed on the RGB color space and spatial ones computed on the HSV color space. On line $i$ and column $j$ of the confusion matrix is the percentage of samples that belong to class $i$ according to 


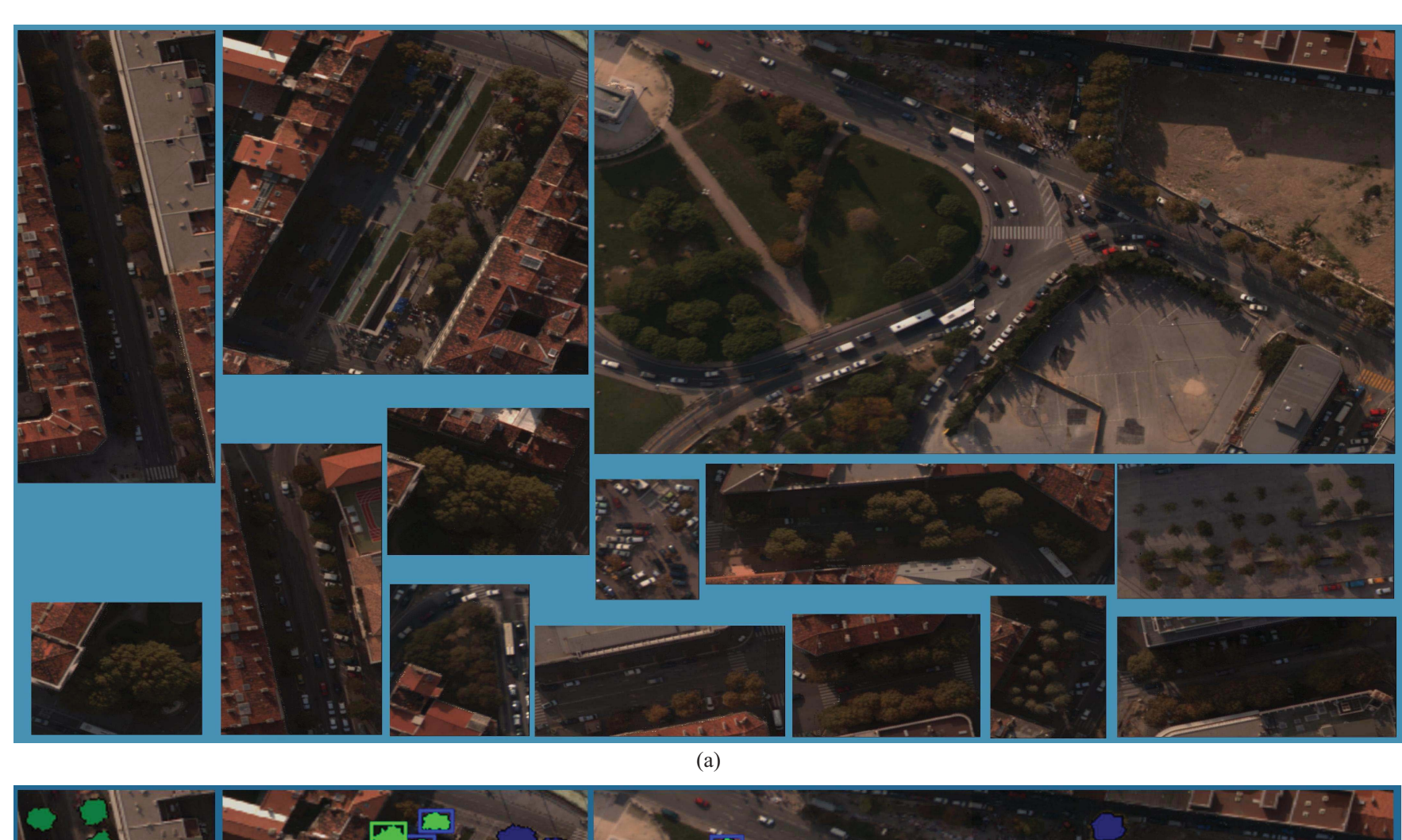

(a)

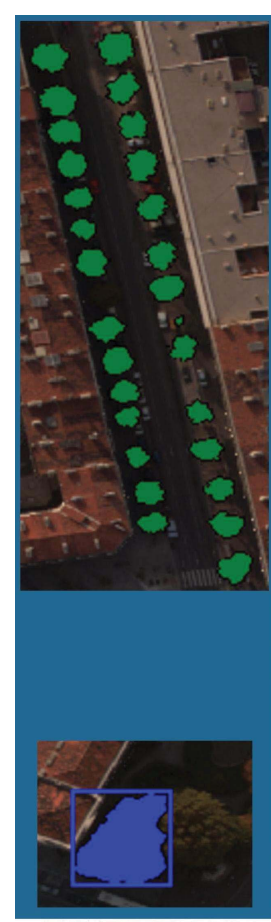

train test

\section{$\square$ Platanus sp.}

- Sophora japonica L.
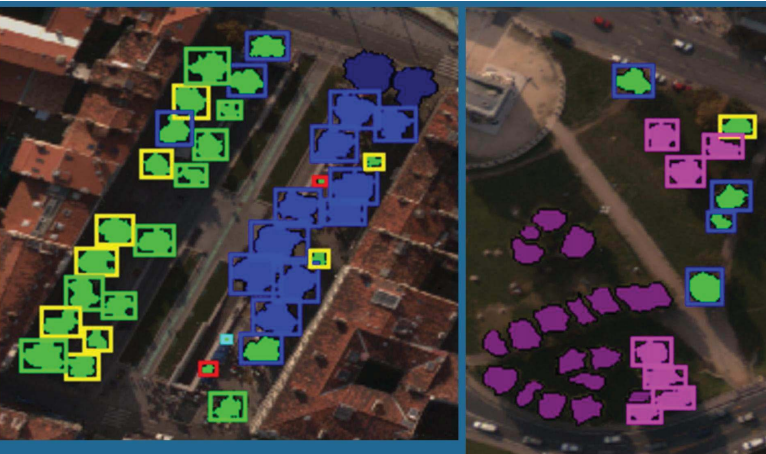
TABLE IV

Overall Accuracy (\%) FOr Spectral and TeXture Feature Vectors COMPUTED ON DIFFERENT COLOR SPACES

\begin{tabular}{|c|c|c|c|c|}
\hline $\begin{array}{ll}\text { Spectral } & \text { Texture }\end{array}$ & RGB & HSV & XYZ & Lab \\
\hline RGB & 65.5 & 76.2 & 65.7 & 69.3 \\
\hline HSV & 69.7 & 73.2 & 67.8 & 73 \\
\hline XYZ & 67.9 & 74.8 & $\begin{array}{l}65.2 \\
\end{array}$ & 61.1 \\
\hline $\mathrm{Lab}$ & 70.6 & 73.9 & 68.8 & 68 \\
\hline
\end{tabular}

TABLE V

Confusion Matrix for TRee Species Classification

\begin{tabular}{|c|c|c|c|c|c|c|c|}
\hline \multicolumn{8}{|c|}{ Ground Truth } \\
\hline & & Platanus & Sophora & Tilia & Celtis & Pinus & Cupressus \\
\hline \multirow{6}{*}{ ธี } & Platanus & 100 & $\overline{0}$ & 19.5 & 28.6 & 0 & $\overline{0}$ \\
\hline & Sophora & 0 & 93.8 & 9.76 & 0 & 0 & 0 \\
\hline & Tilia & 0 & 6.25 & 26.8 & 7.14 & 0 & 0 \\
\hline & Celtis & 0 & 0 & 36.6 & 64.3 & 5.56 & 11.1 \\
\hline & Pinus & 0 & 0 & 2.44 & 0 & 83.3 & 0 \\
\hline & Cupressus & 0 & 0 & 4.88 & 0 & 11.1 & 88.9 \\
\hline
\end{tabular}

results for tree species classification provide initial proofs that spectral and spatial feature vectors can accurately differentiate between tree species belonging to the same taxonomic group. Spectral characteristics are very reliable in separating between taxonomic classes, i.e., deciduous from coniferous tree species. Adding spatial characteristics computed from tree crown regions improves the separation abilities of the SVM classifier for trees belonging to the same taxonomic group.

Tree species information and 3-D tree parameters estimated from aerial imagery are used in Section IV to parametrize by inference an architectural model of tree growth, which is detailed in Section III.

\section{Architectural Model of Tree Growth}

Tree architecture can be seen as the result of the complex interaction of two processes, structural development (based on organogenesis), and functional growth (biomass accumulation and distribution, in interaction with the environment) [10], [28]. Functional-structural models of tree growth aim at dynamically describing these two processes. In the following, we use a derivation of the GreenLab model [29]. Important concepts of the model are presented hereafter.

\section{A. Tree Structural Development}

Trees develop rhythmically by the successive productions of architectural growth units by buds. A growth unit is composed of a set of phytomers, considered as the elementary botanical units of constant composition (generally an internode, a leaf and potential axillary buds). A growth cycle is defined as the time elapsing between the appearances of two growth units [30], [31].

Since plant development can be seen as the result of bud functioning, parallel generative grammars (L-Systems) describing the production of buds have shown to be particularly adapted to model mathematically plant development [32]-[34].
The botanical concept of physiological age (PA) [31] helps differentiate between the different types of axes (and accordingly the organs they are composed of) according to some morphological properties. The main trunk has a physiological age 1 and the oldest physiological age (denoted $P$ ) is the ultimate state of differentiation for an axis, it is usually short, without branches. Usually, less than five physiological ages are necessary to describe the axis typology in trees. Such characterization was recently used in [30] to define the OL-System of the GreenLab model of plant development, as follows.

1) OL-System for GreenLab Organogenesis: A OL-System [35] is defined by an alphabet, an axiom (or seed) and production rules.

a) GreenLab alphabet $\mathcal{G}$ is given by the set of phytomers $\mathcal{M}$ and buds $\mathcal{B}$. A phytomer is defined with four indices: its chronological age: $n$, its physiological age $p$, the physiological age of its axillary buds: $q,(q \geq p)$, the chronological age of the plant $t$. It is denoted by $m_{\mathrm{pq}}^{t}(n)$. Note that considering the temporal indices $(t$ and $n)$ is necessary for the functional growth, since the physical characteristics of the phytomers (masses, dimensions) will vary with time. A bud is simply defined with its physiological age $p$ and plant chronological age $t$, it is denoted by $b_{p}^{t}$. The organogenesis alphabet is given by

$$
\mathcal{G}=\mathcal{M} \cup \mathcal{B}
$$

with

$$
\mathcal{M}=\left\{m_{\mathrm{pq}}^{t}(n)\right\}_{\{1 \leq p \leq P, p \leq q \leq P, 1 \leq t \leq T, 1 \leq n \leq t\}}
$$

and

$$
\mathcal{B}=\left\{b_{p}^{t}\right\}_{1 \leq p \leq P, 0 \leq t \leq T}
$$

$T$ denotes the maximum time of the simulation. No symbols are considered for organs as the constitution of a phytomer is supposed fixed by botanical rules:

b) the seed is a bud of physiological age $1 b_{1}^{0}$;

c) GreenLab production rules $P_{r}$ are given by

For all $(p, t) \in[1 ; P] \times[0 ; T-1]$

$$
b_{p}^{t} \longrightarrow\left(\prod_{q=p}^{P}\left(m_{\mathrm{pq}}^{t+1}(1)\right)^{u_{\mathrm{pq}}(t+1)}\left(b_{q}^{t+1}\right)^{v_{\mathrm{pq}}(t+1)}\right) b_{p}^{t+1}
$$

and for all $(t, n, p, q) \in[1 ; T-1] \times[1 ; t] \times[1 ; P] \times$ $[p ; P]$

$$
m_{\mathrm{pq}}^{t}(n) \longrightarrow m_{\mathrm{pq}}^{t+1}(n+1)
$$

with

1) $u_{\mathrm{pq}}(t)$ : number of phytomers $m_{\mathrm{pq}}$ in a growth unit of PA $p$, appearing at growth cycle $t$, and

2) $v_{\mathrm{pq}}(t)$ : number of active axillary buds of PA $q$ in a growth unit of PA $p$, appearing at growth cycle $t$.

$\left(u_{\mathrm{pq}}(t)\right)_{t}$ and $\left(v_{\mathrm{pq}}(t)\right)_{t}$ are the development sequences of the model. 
2) Structural Factorization: A fundamental result (or rather hint) given by [32] is the ability to factorize the L-system productions. It showed particularly adapted to the concept of physiological age, plant structure can be factorized into smaller parts that may repeat themselves a large number of times.

Definition 1 (Substructure): At growth cycle $t \geq 0$, a substructure of physiological age $p, 1 \leq p \leq P$ and chronological age $n, 0 \leq n \leq t$ is a word on the alphabet $\mathcal{G}$ defined as the complete plant structure that is generated after $n$ cycles by a bud of physiological age $p$. It is denoted by $S_{p}^{t}(n)$ (corresponding to the structure generated by $b_{p}^{t-n}$ after $n$ growth cycles).

Substructures of chronological age 0 are buds. The fundamental property is that in the deterministic case, at a given growth cycle, all the substructures with the same physiological and chronological ages are identical.

By iterating the production rules, the following important result is deduced in [30].

Theorem 1 (Dynamic Equation of Plant Development): For all $t \geq 1, n \in[1, t], p \in[1, P], q \in[p, P]$, we have

$$
S_{p}^{t}(n)=R_{p}^{t}(n) S_{p}^{t}(n-1)
$$

with

$$
R_{p}^{t}(n)=\left[\prod_{p \leq q \leq P}\left(m_{\mathrm{pq}}^{t}(n)\right)^{u_{\mathrm{pq}}(t+1-n)}\left(S_{q}^{t}(n-1)\right)^{v_{\mathrm{pq}}(t+1-n)}\right]
$$

In (6), substructure $S_{p}^{t}(n)$ is decomposed into

1) its oldest growth unit, called base growth unit

$$
\prod_{p \leq q \leq P}\left(m_{\mathrm{pq}}^{t}(n)\right)^{u_{\mathrm{pq}}(t+1-n)}
$$

2) the lateral substructures borne by the base growth unit (they are one cycle younger)

$$
\prod_{p \leq q \leq P}\left(S_{q}^{t}(n-1)\right)^{v_{\mathrm{pq}}(t+1-n)}
$$

3) the substructure grown from the apical bud of the base growth unit (also one cycle younger) $S_{p}^{t}(n-1)$.

Substructures and phytomers will be repeated a lot of times in the tree architecture, but they need to be computed only once for each kind, by a recursive construction [30].

\section{B. Functional Growth in Interaction With the Environment}

Biomass production and allocation in GreenLab are described with a source-sink model [36]. Biomass production is computed according to Beer-Lambert's law [37] and to the environmental conditions. For an individual tree, the biomass production at growth cycle $t$ denoted $Q(t)$ is given by

$$
Q(t)=E(t) \mu S_{p}(t)\left(1-e^{-k \frac{S(t)}{S_{p}(t)}}\right)
$$

where $E(t)$ is an environmental function at growth cycle $t$ related to light radiation and to hydric stress, $S(t)$ is the tree leaf surface area, $k$ is the extinction coefficient for BeerLambert's law, $\mu$ is the conversion efficiency and $S_{p}(t)$ is a characteristic surface area related to tree crown $x-y$ projection and to the space potentially available for tree development. As proposed in [29], $S_{p}(t)$ is given at growth cycle $t$ by

$$
S_{p}(t)=\min \left[S_{0}\left(\frac{S(t)}{S_{0}}\right)^{\alpha}, S_{d}\right]
$$

with $S_{0}$ and $\alpha$ two empirical coefficients estimated by model inversion from experimental data and $S_{d}$, the surface area of the Voronoi cell which can be associated to the individual tree when considering the Voronoi diagram obtained from the distribution of all tree locations. $S_{p}(n)$ grows with the development of leaf surface and saturates at $S_{d}$.

Biomass is then allocated to the root system, to rings for the secondary growth and to buds for the new growth units proportionally to sink strengths [36], defined as parametric functions. The sum of all sink strengths at growth cycle $t$ defines the plant demand $D(t)$. Along plant growth, the ratio of available biomass to demand $Q(t) / D(t)$ is thus representative of the competition for resources between organs.

All the functional state variables are computed only once for each phytomer and each substructure of each type, and as the number of phytomers and substructures is proportional to plant age, the computation time of a simulation is a quadratic function of the final plant age, and is no longer proportional to the number of organs (which can increase exponentially with the simulation time for complex trees; see [10]). The computation of $S(t)$ and $D(t)$ is done recursively by applying a proper morphism to (6).

\section{Interaction Structure-Function}

A fundamental property of trees is their plasticity in response to changes in their growth conditions. Plants are seen as self-regulating systems that can react to exogenous (e.g., environmental stresses that reduce plant production; organ or branch pruning that reduce plant demand) or endogenous (e.g., apparition of new branches or fruits that increase demand) influences.

To model this plasticity, the ratio of available biomass to demand $(Q / D)$ controls the appearance and longevity of new organs and branches, thus updating the potential structure at each growth cycle. The development sequences $\left(u_{\mathrm{pq}}(t)\right)_{t}$ and $\left(v_{\mathrm{pq}}(t)\right)_{t}$ driving the production rules of the development grammar [see (4)] can be modeled as functions of this ratio, for example

$$
\left\{\begin{array}{l}
u_{\mathrm{pq}}(t)=\left\lfloor A_{\mathrm{pq}}^{1}+A_{\mathrm{pq}}^{2} \cdot \frac{Q(t-1)}{D(t-1)}\right\rfloor \\
v_{\mathrm{pq}}(t)=\left\lfloor B_{\mathrm{pq}}^{1}+B_{\mathrm{pq}}^{2} \cdot \frac{Q(t-1)}{D(t-1)}\right\rfloor
\end{array}\right.
$$

where for a real number $x,\lfloor x\rfloor$ is its rounded value and $A_{\mathrm{pq}}^{1}$, $A_{\mathrm{pq}}^{2}, B_{\mathrm{pq}}^{1}, B_{\mathrm{pq}}^{2}$ are model parameters. In Fig. 9, are presented the simulation results for trees of the same type and same age growing in different competition conditions. It impacts the production equation (7) via the equation of $S_{p}(t)$ giving the potential space for each tree in (8), thus implying drastic changes in the ratio of available biomass to demand and different architectural development. 

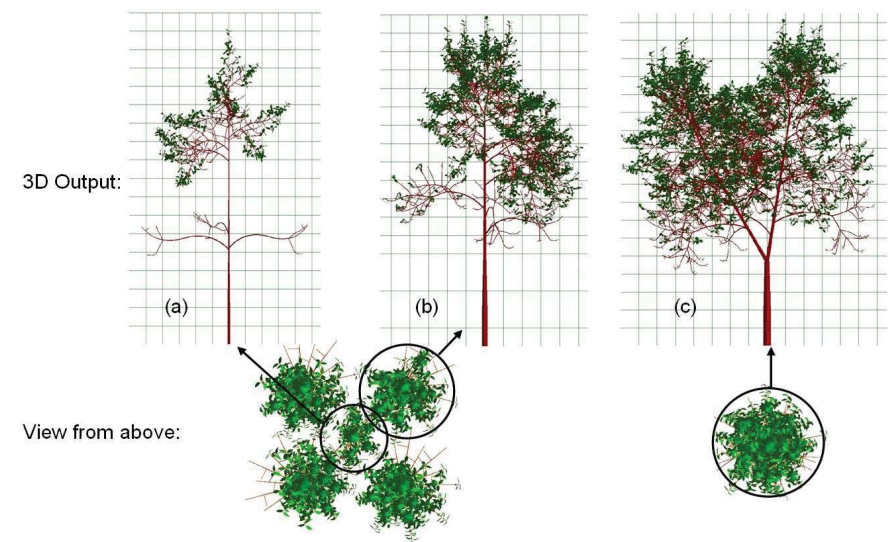

Fig. 9. Simulation of tree growth in heterogeneous conditions: view from above and detailed architectures of individuals. (a) Tree surrounded by four neighbors in a clump severely suffering from competition. (b) Tree on the edge of the clump. (c) Tree growing in open-field like conditions reiterates (a fork appears).

\section{Model Parameterization}

An objective of plant growth models is to help decorrelate genetic and environmental effects in plant growth [38]. Ideally, model parameters driving the structural development and biophysical interaction with the environment should be characteristic of a species, and remain stable in a large range of environmental conditions: "one genotype = one set of parameters". In [39], the same set of GreenLab parameters was used to fit experimental data of beech trees grown in very different environmental conditions. The estimation process described in [39] is, however, quite complex, especially since most parameters are hidden parameters and the collection of detailed enough experimental data on trees is tedious. Therefore, the parameters of only a few species are estimated so far. The other inputs for the model are thus: 1) the environmental conditions $E(t)$ in $(7) ; 2$ ) the potentially available space $S d$ in $(8)$; and 3) plant age.

\section{IMAGE-BASED MODEL PARAMETERIZATION}

In this section, we present the methodology used to parameterize GreenLab tree model with tree variables extracted by means of aerial image analysis.

For a tree $T$ in a given scene to be modeled, we need to characterize all the necessary inputs described in Section III-D: species-specific parameters, environmental conditions, potentially available space (characteristic of the level of competition with neighbours), and plant age. It is thus necessary that parameters of the species identified by image analysis Section II are available.

For the identified species, we will assume that the parameterization is performed in other field studies, and that the species-specific parameters are available. The objective is thus to estimate plant age $N$, the environmental conditions (supposed as an average constant value $E$ ) and the potentially available space $S_{d}$. The latter can be directly deduced by computing the Voronoi diagram of the scene from tree locations. For $N$ and $E$, we simply use a least-square estimator. Tree height and crown diameter are model outputs (tree geometry is simply obtained from tree structure and organ dimensions with some geometrical rules). We thus minimize

$$
J(E, N)=(\tilde{h}(E, N)-h)^{2}+(\tilde{c d}(E, N)-c d)^{2}
$$

in which $\tilde{h}$ and $\tilde{c d}$ are computed with the model, and $h, c d$ are the height and crown diameter estimated from aerial imagery. Mostly because of tree plasticity, the function is nonconvex, but can be minimized with simple heuristics like simulated annealing. Note that the retroaction of functioning on structure development is a key process of the model that allows a proper exploration of the real state space. Another advantage of the GreenLab model for this paper is that the structural factorization induces a very efficient growth simulation, which makes it possible to perform the minimization. It would be prohibitive without the grammar factorization. Finally, some prior knowledge can be used, like tree age (often available in urban environments or in plantations), or specific dendrometric relationships (linking age, environment, tree shape) in forestry literature.

Individual simulations with GreenLab for all trees in the scene provides architectural and geometrical variables, at organ scale. It is thus possible to obtain realistic 3-D rendering for each tree. After simulation, each tree is described through a high-level format, based on the AMAP technology [40]. It represents a tree through an organ list, each with its associated location and orientations. The data thus produced can be used for very detailed scene visualization. For quick visualization purposes, large-scale scenes or real-time applications, simple billboarding is preferred to illustrate experimental results.

\section{Results AND Evaluation}

Results of architectural tree growth model parameterization obtained from image analysis are presented in the following. Heights $h$, crown diameters $c d$, positions, and species are extracted from aerial data for a population of trees in an urban environment. Only one species is represented in this set (Platanus) but with some diversity in crown sizes and heights. Fig. 10 shows billboard representations of the functional tree models automatically inserted in the corresponding 3-D city model according to location parameters estimated for each tree as the barycenter of the tree crown.

Fig. 10(a) shows a part of the downtown Marseille, France, consisting of several trees of different crown sizes and heights. Trees are represented by billboards obtained from screenshots of the functional tree models parameterized to fit tree crown area and tree height estimated for each tree crown by image analysis.

Tree billboards are automatically inserted into the 3-D city model according to location parameters extracted for each tree. The accuracy of the location parameters for the trees can be visually verified. The interest of having used a functional model of plant growth for 3-D reconstruction is that it allows to see the evolution of the tree populations across years. Fig. 10(b) shows the appearance of urban vegetation in the same scene as in Fig. 10(a) after a period of five years and under the assumption that no branch pruning was performed during the elapsed time. 


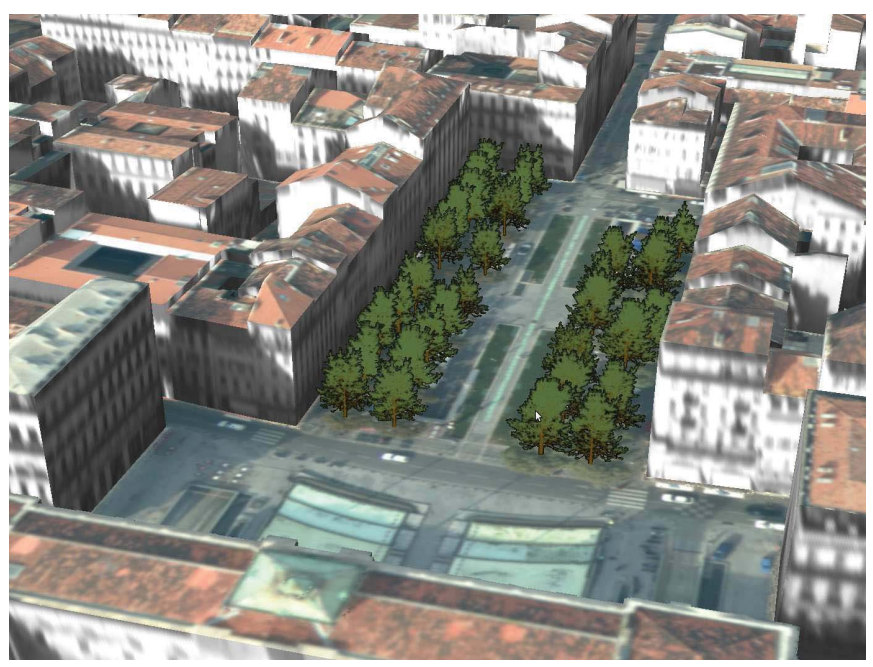

(a)

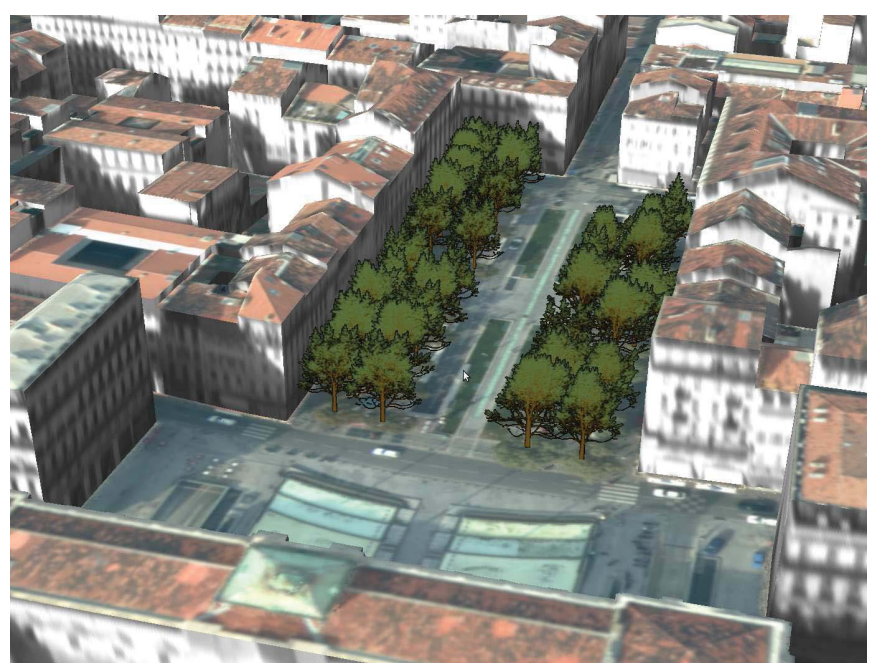

(b)

Fig. 10. Three-dimensional city model including functional vegetation models. (a) Trees are represented by billboards obtained from screenshots of the functional tree models exploiting tree height and crown areas estimated for each tree crown. (b) 3-D city model presenting the time evolution of the vegetation cover including five-year older vegetation models for the reconstructed area.

\section{CONCLUSION}

We presented a complete and novel approach for the automatic reconstruction of natural environments from aerial imagery data. Unlike existing techniques, the vegetation component of the environment was modeled by functionalstructural individual-based models of tree growth. To achieve this, aerial data was analyzed and vegetation characteristics estimated were used to generate individual-based models of tree growth. Experimental results were presented, which verify the validity of our approach that can successfully be applied to different 3-D virtual environments. Finally, we presented the time evolution simulation of the generated 3-D individual plant-growth models within the 3 -D environment.

Linking the recent progresses in both vegetation analysis from aerial images and vegetation functional-structural modeling offers interesting perspectives for the reconstruction of both urban or natural environments, with potential applications offered by the possibility of simulating the future evolution of plant populations.

The process of species-specific functional parameter estimation is very tedious as mentioned in [41]. Therefore, it is very difficult to get a priori a large data-base of species for an automatic reconstruction of a city for example, in which gardeners usually endeavored to find the most original species of trees. It is easier to reconstruct tree plantations or even natural forests of temperate regions (in which the diversity of species is usually quite limited). It is also true that the functional reconstruction (that is to say with the proper biophysical parameters of trees) is more crucial for forestry applications, to predict wood production or to optimize forest management than for the 3-D reconstruction of cities.

As spatial resolution of remotely sensed data increases, the methodology used for aerial data analysis could be applied on other types of sensors, such as satellite [42] or light detection and ranging [43] data. Experimental results reported in this paper were applied on a urban environment test-case but the methodology can be extended to other types of natural environments, such as forest [44] landscapes.

\section{ACKNOWLEDGMENT}

The authors would like to thank the French National Survey Agency (IGN) for kindly providing the remote sensing data used in this paper.

\section{REFERENCES}

[1] R. Mech and P. Prusinkiewicz, "Visual models of plant interacting with their environment," in Proc. ACM 23rd Annu. Conf. Comput. Graph. Interact. Tech., 1996, pp. 397-410.

[2] O. Deussen and B. Lintermann, Digital Design of Nature-Computer Generated Plants and Organics. New York, NY, USA: Springer-Verlag, 2005.

[3] U. Nations. (2008). The United Nations Collaborative Programme on Reducing Emissions from Deforestation and Forest Degradation in Countries [Online]. Available: http://www.unredd.net/index.php? option=com_docman \&task=doc_download $\&$ gid $\%=4598 \&$ Itemid $=53$

[4] H. Gibbs, S. Brown, J. Niles, and J. Foley, "Monitoring and estimating tropical forest carbon stocks: Making REDD a reality," Environ. Res. Lett., vol. 2, no. 4, p. 045023, 2007.

[5] S. Goetz, A. Baccini, N. Laporte, T. Johns, W. Walker, J. Kellndorfer, R. Houghton, and M. Sun, "Mapping and monitoring carbon stocks with satellite observations: A comparison of methods," Carbon Balance Manage., vol. 4, no. 1, p. 2, 2009.

[6] C. Varekamp and D. Hoekman, "High-resolution InSAR image simulation for forest canopies," IEEE Trans. Geosci. Remote Sens., vol. 40, no. 7, pp. 1648-1655, Jul. 2002.

[7] Z. Guo, G. Sun, and Z. Zhang, "A three-dimensional radar backscatter model for larch forest using L-system," in Proc. IEEE Int. Geosci. Remote Sens. Symp., vol. 5. Jul. 2003, pp. 3245-3247.

[8] D. Liu, G. Sun, Z. Guo, K. Ranson, and Y. Du, "Three-dimensional coherent radar backscatter model and simulations of scattering phase center of forest canopies," IEEE Trans. Geosci. Remote Sens., vol. 48, no. 1, pp. 349-357, Jan. 2010.

[9] C. Iovan, D. Boldo, and M. Cord, "Detection, characterization and modeling vegetation in urban areas from high resolution aerial imagery," IEEE J. Sel. Topics Appl. Earth Observat. Remote Sens., vol. 1, no. 3, pp. 206-213, Sep. 2008.

[10] R. Sievänen, E. Nikinmaa, P. Nygren, H. Ozier-Lafontaine, J. Perttunen, and H. Hakula, "Components of a functional-structural tree model," Ann. Forest Sci., vol. 57, nos. 5-6, pp. 399-412, 2000.

[11] C. Thom and J.-P. Souchon, "The IGN digital camera system in progress," in Photogrammetric Week, vol. 99, R. Fritsch and D. Spiller, Eds. Heidelberg, Germany: Wichmann Verlag, 1999, pp. 89-94. 
[12] N. Paparoditis, J.-P. Souchon, G. Martinoty, and M. Pierrot-Deseilligny, "High-end aerial digital cameras and their impact on the automation and quality of the production workflow," ISPRS J. Photogram. Remote Sens., vol. 60 , no. 6 , pp. 400-412, 2006.

[13] T. M. Lillesand, R. W. Kiefer, and J. W. Chipman, Remote Sensing and Image Interpretation, 6th ed., N. Hoboken, Ed. New York, NY, USA: Wiley, 2008

[14] N. Champion and D. Boldo, "A robust algorithm for estimating digital terrain models from digital surface models in dense urban areas," in Proc. ISPRS Commission 3 Symp., Photogram. Computer Vis., 2006, pp. 1-6.

[15] P. Gong, R. Pu, G. S. Biging, and M. R. Larrieu, "Estimation of forest leaf area index using vegetation indices derived from hyperion hyperspectral data," IEEE Trans. Geosci. Remote Sens., vol. 41, no. 6, pp. 1355-1362, Jun. 2003.

[16] A. Bannari, D. Morin, and D.-C. He, "Caractérisation de lenvironnement urbain à laide des indices de végétation dérivés à partir des données de hautes résolutions spatiale et spectrale," in Télédétection des Milieux Urbains et Périurbains : Sixièmes Journées Scientifiques du Réseau Télédétection de IAUPELF-UREF (Actualit scientifique), L.-M. M. Dubois and P. Lafrance, Eds. Lige, Paris: John Libbey Eurotext, Oct. 1995, pp. 47-64.

[17] B. E. Boser, I. M. Guyon, and V. N. Vapnik, "A training algorithm for optimal margin classifiers," in Proc. 5th Annu. Workshop Comput. Learn. Theory, 1992, pp. 144-152.

[18] C.-C. Chang and C.-J. Lin. (2001). LIBSVM [Online]. Available: http://www.csie.ntu.edu.tw/ cjlin/libsvm

[19] G. Perrin, X. Descombes, and J. Zerubia, "A marked point process model for tree crown extraction in plantations," in Proc. IEEE Int. Conf. Image Process., Sep. 2005, pp. 661-664.

[20] M. Erikson and K. Olofsson, "Comparison of three individual tree crown detection methods," Mach. Vis. Appl., vol. 16, no. 4, pp. 258-265, 2005.

[21] D. Culvenor, "Extracting individual tree information: A survey of techniques for high spatial resolution imagery," in Remote Sensing of Forest Environments: Concepts and Case Studies. Norwell, MA, USA: Kluwer, 2003, pp. 255-278.

[22] J. Secord and A. Zakhor, "Tree detection in aerial lidar and image data," in Proc. Int. Conf. Image Process., Atlanta, GA, USA, Sep. 2006, pp. $2317-2320$.

[23] P. Horváth, I. H. Jermyn, Z. Kato, and J. Zerubia, "A higher-order active contour model for tree detection," in Proc. Int. Conf. Pattern Recognit., Aug. 2006, pp. 130-133.

[24] L. Vincent and P. Soille, "Watersheds in digital spaces: An efficient algorithm based on immersion simulations," IEEE Trans. Pattern Anal. Mach. Intell., vol. 13, no. 6, pp. 583-598, Jun. 1991.

[25] R. M. Haralick, K. Shanmugam, and I. H. Dinstein, "Textural features for image classification," IEEE Trans. Syst., Man Cybern., vol. 3, no. 6, pp. 610-621, Nov. 1973.

[26] P. Ceccato, S. Flasse, S. Tarantola, S. Jacquemoud, and J. M. Gregoire, "Detecting vegetation leaf water content using reflectance in the optical domain," Remote Sens. Environ., vol. 77, no. 1, pp. 22-33, 2001.

[27] C.-W. Hsu and C.-J. Lin, "A comparison of methods for multi-class support vector machines," IEEE Trans. Neural Netw., vol. 13, no. 2, pp. 415-425, Mar. 2002.

[28] A. Mathieu, P.-H. Cournède, V. Letort, D. Barthélémy, and P. de Reffye, "A dynamic model of plant growth with interactions between development and functional mechanisms to study plant structural plasticity related to trophic competition," Ann. Botany, vol. 103, no. 8, pp. 1173-1186, 2009.

[29] P.-H. Cournède, A. Mathieu, F. Houllier, D. Barthélémy, and P. de Reffye, "Computing competition for light in the GREENLAB model of plant growth: A contribution to the study of the effects of density on resource acquisition and architectural development," Ann. Botany, vol. 101, no. 8, pp. 1207-1219, 2008.

[30] P.-H. Cournède, M.-Z. Kang, A. Mathieu, J.-F. Barczi, H.-P. Yan, B.G. Hu, and P. de Reffye, "Structural factorization of plants to compute their functional and architectural growth," Simulation, vol. 82, no. 7, pp. $427-438,2006$.

[31] D. Barthélémy and Y. Caraglio, "Plant architecture: A dynamic, multilevel and comprehensive approach to plant form, structure and ontogeny," Ann. Botany, vol. 99, no. 3, pp. 375-407, 2007.

[32] A. Smith, "Plants, fractals and formal languages," ACM SIGGRAPH Computer Graph., vol. 18, no. 3, pp. 1-10, 1984.

[33] P. Prusinkiewicz, A. Lindenmayer, and J. Hanan, "Developmental models of herbaceous plants for computer imagery purposes," Comput. Graph., vol. 22, no. 4, pp. 141-150, 1988.
[34] W. Kurth, Growth Grammar Interpreter GROGRA 2.4-A Software Tool for the 3-Dimensional Interpretation of Stochastic, Sensitive Growth Grammars in the Context of Plant Modelling. Introduction and Reference Manual. Gottingen Germany: Selbstverl des Forschungszentrums Waldökosysteme der Universität Göttingen, 1994.

[35] A. Lindenmayer, "Mathematical models for cellular interactions in development. I. Filaments with one-sided inputs," J. Theoretical Biol., vol. 18, no. 3, pp. 280-289, 1968.

[36] J. Warren-Wilson, "Ecological data on dry matter production by plants and plant communities," in The Colloection and Processing of Field Data, E. Bradley and O. Denmead, Eds. New York, NY. USA: Interscience, 1967, pp. 77-123.

[37] J. Vose, N. Sullivan, B. Clinton, and P. Bolstad, "Vertical leaf area distribution, light transmittance, and application of the beer-lambert law in four mature hardwood stands in the southern Appalachians," Can. J. Forest Res., vol. 25, no. 6, pp. 1036-1043, 1995.

[38] G. Hammer, M. Cooper, F. Tardieu, S. Welch, B. Walsh, F. Van Eeuwijk, S. Chapman, and D. Podlich, "Models for navigating biological complexity in breeding improved crop plants," Trends Plant Sc, vol. 11, no. 12 , pp. $587-593,2006$

[39] V. Letort, P.-H. Cournède, A. Mathieu, P. de Reffye, and T. Constant, "Parametric identification of a functional-structural tree growth model and application to beech trees (Fagus sylvatica)," Funct. Plant Biol., vol. 35, no. 10, pp. 951-963, 2008.

[40] M. Jaeger and P. de Reffye, "Basic concepts of computer simulation of plant growth," J. Biosci., vol. 17, no. 3, pp. 275-291, Sep. 1992.

[41] P.-H. Cournède, V. Letort, A. Mathieu, M.-Z. Kang, S. Lemaire, S. Trevezas, F. Houllier, and P. de Reffye, "Some parameter estimation issues in functional-structural plant modelling," Math. Model. Natural Phenomena, vol. 6, no. 2, pp. 133-159, 2011.

[42] Y. O. Ouma and R. Tateishi, "Urban-trees extraction from QuickBird imagery using multiscale spectex-filtering and non-parametric classification," ISPRS J. Photogram. Remote Sens., vol. 63, no. 3, pp. 333-351, 2008.

[43] L. Changok, "Estimation of urban tree crown volume based on object-oriented approach and lidar data," M.S. thesis, Faculty of GeoInformation Science and Earth Observation, ITC Enschede, Enschede, The Netherlands, 2007.

[44] M. Erikson, G. Perrin, X. Descombes, and J. Zerubia, "A comparative study of three methods for identifying individual tree crowns in aerial images covering different types of forests," in Proc. Int. Soc. Photogram. Remote Sens., 2006, pp. 1-5.

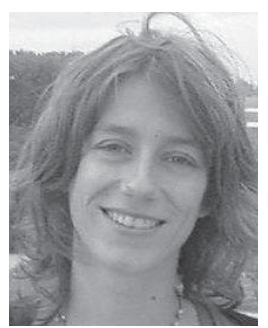

Corina Iovan received the Ph.D. degree in computer science from the Université Pierre et Marie CurieParis 6, Paris, France, in 2009. During her Ph.D. studies with the French National Survey Agency (IGN), she specialized in tree species characterization from aerial imagery.

She joined the French Institute for Research in Information and Communication Science and Technologies (INRIA Saclay - Île-de-France) and Ecole Centrale de Paris as a Post-Doctoral Fellow. Her current research interests include data mining/machine learning applied to big datasets, remote sensing, and computer vision.

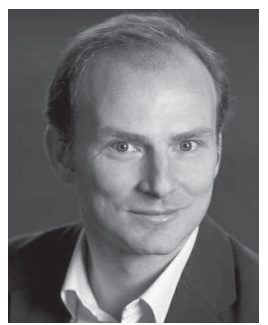

Paul-Henry Cournède is a Professor in applied mathematics with the Ecole Centrale Paris and the Head of the Research Team Digiplante, Laboratory of Applied Mathematics and Systems, Paris, France.

His current research interests include mathematical modeling of plant growth, and parameter estimation and optimization. 


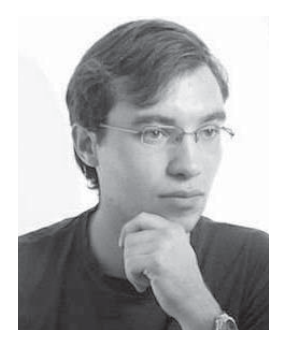

Thomas Guyard is a Software Engineer with the Digiplante Team, INRIA Saclay - Île-de-France, and has co-developed several modeling, simulation, and visualization platforms for plant growth.

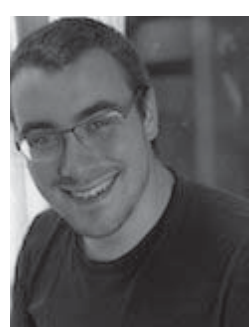

Benoît Bayol is a Software Engineer with the Digiplante Team, INRIA Saclay - Île-de-France, and has co-developed several modeling, simulation, and visualization platforms for plant growth.

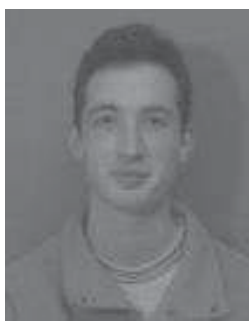

Didier Boldo graduated from Ecole Polytechnique in 1996 and from Ecole Nationale des Sciences Géographiques in 1998. He received the Ph.D. degree from the René Descartes University, Paris, France.

$\mathrm{He}$ is currently a Researcher with Electricité de France R\&D. Prior to this, he was the Head of MATIS Laboratory, French National Survey Agency from 1998 to 2009. His current research interests include image and signal processing. His principal research is 3-D reconstruction of manmade objects and metrology using images (photogrammetry).

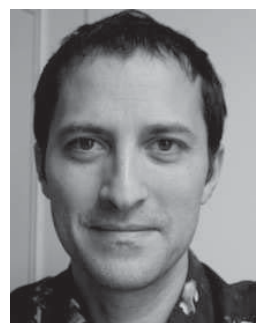

Matthieu Cord received the Ph.D. degree in computer science from the University UCP, Cergy- Pontoise cedex, France, in 1998, before working with the ESAT Lab, KUL University, Leuven, Belgium, and in the ETIS Lab, Cergy-Pontoise cedex, as an Assistant Professor.

$\mathrm{He}$ is currently a Full Professor of computer science with UPMC Paris 6 Sorbonne Universities. In 2009, he was nominated at the IUF (French Research Institute) for a five-year delegation position. $\mathrm{He}$ developed several systems for content-based image and video retrieval, focusing on interactive learning-based approaches. He is also interested in machine learning for multimedia processing, digital preservation, and Web archiving. He has authored 100 scientific publications and participated in several international projects (European FP6 and FP7, Singapore, Brazil) in his areas of expertise. His current research interests include computer vision, image processing, and pattern recognition. 